\title{
ON BOUNDARY VALUE PROBLEM FOR EQUATIONS WITH CUBIC NONLINEARITY AND STEP-WISE COEFFICIENT
}

\author{
A. KiRICHUKA AND F. SADYRBAEV
}

Abstract. The differential equation with cubic nonlinearity $x^{\prime \prime}=-a x+b x^{3}$ is considered together with the boundary conditions $x(-1)=x(1)=0$. In the autonomous case, $b=$ const $>0$, the exact number of solutions for the boundary value problem is given. For nonautonomous case, where $b=\beta(t)$ is a step-wise function, the existence of additional solutions is detected. The reasons for such behaviour are revealed. The example considered in this paper is supplemented by a number of visualizations.

Mathematics subject classification (2010): 34B15, 34B05, 34C10.

Keywords and phrases: Boundary value problem, step-wise function, cubic nonlinearity, phase trajectory, multiplicity of solutions, Jacobian elliptic function.

\section{REFERENCES}

[1] E. Ellero AND F. ZANolin, Homoclinic and heteroclinic solutions for a class of second-order nonautonomous ordinary differential equations: multiplicity results for stepwise potentials, Boundary Value Problems 2013:167, (2013), 167.

[2] A. Gritsans, F. Sadyrbaev, Extension of the example by Moore-Nehari, Tatra Mt. Math. Publ. 63, (2015), 115-127.

[3] M. W. Hirsch, S. Smale, R. L. Devaney, Differential equations, dynnamical systems, and an introduction to chaos, Academic Press Elsevier, 2004.

[4] A. KIRICHUKA, Multiple solutions for nonlinear boundary value problems of ODE depending on two parameters, Proceedings of IMCS of University of Latvia 13, (2013), 83-97.

[5] A. KIRICHUKA, Multiple Solutions of Nonlinear Boundary-value Problems for Ordinary Differential Equations, Journal of Mathematical Sciences 6, 205(2015), 768-776.

[6] A. KiRICHUKA, On the Dirichlet boundary value problem for a cubic on two outer intervals and linear in the internal interval differential equation, Proceedings of IMCS of University of Latvia 16, (2016), 54-66.

[7] N. B. Konyukhova, A. A. Sheina, On an Auxiliary Nonlinear Boundary Value Problem in the Ginzburg-Landau Theory of Superconductivity and its Multiple Solution, FRC CSC of RAS, Moscow, Russia 3, (2016), 5-20.

[8] R. MOORE AND Z. NeHARI, Nonoscillation theorems for a class of nonlinear differential equations, Trans. Amer. Math. Soc. 93, (1959), 30-52.

[9] S. OGORODNIKOVA, F. SADYRBAEV, Multiple solutions of nonlinear boundary value problems with oscillatory solutions, Mathematical Modelling and Analysis 4, 11(2006), 413-426.

[10] E. T. Whittaker And G. N. Watson, A Course of Modern Analysis, Cambridge University Press, ISBN 0-521-58807-3, 1940, 1996. 